\title{
Performance of DWT and SWT in muscle fatigue detection
}

\begin{abstract}
Ability of wavelet transform in accessing time and frequency information at the same time make it widely used in analyzing bio-signals like electromyography (EMG). Discrete wavelet transforms (DWT) and stationary wavelet transform (SWT) are examples of analysis based on wavelet. Both analyses are based on decomposition technique and splitting signals into few frequency band. The different is DWT will down sample resolution into half at each decomposition level, while SWT is not. This paper is investigating both analyses in its ability on de-noising process of EMG using the same properties. The signals will be decomposed into five level of decomposition using 'db20', and de-noised using the same threshold setting. The performance will be evaluated based on its signals to noise ratio and muscle fatigue detection. Results show that de-noising process through SWT give better signals to ratio. Inability in DWT removed $20 \mathrm{~Hz}$ corner frequency in several reading lead to misinterpretation in fatigue detection.
\end{abstract}

Keyword: De-noising; DWT; Electromyograhy; Muscle fatigue; SWT 